\title{
The case for a great many journals
}

\author{
General opinion reacts badly against new journals, but on the grounds that there are too many already. But any issue of \\ any journal contains something of more than passing interest.
}

WHY are there so many journals? The simple answer is that there are so many people working at research, all of whom must publish regularly, so that there is an endless demand for pages that will carry text. And because there are limits to the growth of individual journals, perhaps determined by economic considerations or even by editors' proper sympathy for the amount of material their readers can handle in one package, it follows that there must be a secular increase in the number of separate journals.

That is one side of the coin. The other is that, however far down in the peckingorder of selectivity a journal may be, it is very rare that its consecutive issues fail to include something of wide general interest. Inveterate browsers in the libraries know that already. What follows is an account of one browser's findings in a handful of journal issues reaching this office on a recent day, and selected (without knowledge of the purpose) by somebody else.

First, there is Physics Letters A, which happens to include a discussion of an argument originally put up by Per Bak and K. Sneppen (Phys. Rev. Lett. 71, 4087$4090 ; 1993)$ to the effect that the episodic character of the fossil record, called punctuated equilibrium by S. J, Gould, may be explicable by a simple mathematical model. The model (see Nature 371, 197; 1994) supposes a one-dimensional lattice and the assignment to each vertex of a random real number (between 0 and 1 ).

Each point represents a species and its random number its fitness. The "ecosystem" then evolves by the simple rule that the smallest number in the chain and its two nearest neighbours are replaced by another random number in the chain. This rule creates a bias against small numbers; if the lattice is infinite, they will all grow to be bigger than some critical limit. But in that condition, the lattice is unstable. If, at any step, the smallest number and its two neighbours should by chance be replaced by numbers less than the critical value, there will be an avalanche of small numbers, or an extinction.

Peter Grassberger from the University of Wuppertal (Phys. Lett. A 200, 277-282; 1995) now says that it seems "very unlikely" to him that such a static model could represent a real ecosystem; in another context, he uses the slighting phrase "a physicist's toy model". He nevertheless suspects that it may be a model that "sooner or later" will find an application. $\mathrm{He}$ even considers the question of whether the model is a special case of the model system known as "directed percolation", originally devised to account for collisions between hadrons, but now more properly applicable to epidemics and the spreading of damage. He concludes that it is not. So the question remains as to what the model really applies.

Then there is the matter of the claimed new allotrope of carbon ("white carbon"?), prepared by Sei-ichi Tanuma and Andrei Palnichenko at the Iwaki Meisei University in Japan (J. Mater. Res. 10, $1120-1125$; 1995). Already, of course, there are diamond and graphite, not to mention $\mathrm{C}_{60^{\circ}}$. Crystals of the new material are made by condensing carbon vapour to room temperature, and form either rhombohedral or hexagonal crystals (up to a millimetre across) with their principal axes perpendicular to the substrate. They are fibrous, and white in colour, with a density of $1.46 \mathrm{~g} \mathrm{~cm}^{-3}$, whence the etymologically careless name of "carbolite".

The explanation? Think of a string of carbon atoms held together by double bonds (called cumulene), perhaps made by the dehydrogenation of acetylene. This is one-dimensional carbon, or carbyne. Then stack these molecules parallel to each other and you have a material that forms a crystal held together by lateral van der Waals forces between the chains. The chains themselves are staggered, with four atoms in a line followed by four offset by a small degree. The interchain separation is $0.344 \mathrm{~nm}$ in the hexagonal crystal and $0.337 \mathrm{~nm}$ in the rhombohedral variety. It is a good tale that will be more persuasive when there is an X-ray structure.

Now AIDS in Thailand. This week's Lancet carries a study of the degree to which couples in which the male is infected with HIV carry similar serological types of the virus. Thailand stands out in the annals of the AIDS epidemic by the speed with which the disease has spread in the past few years. Dr Chaiyos Kunanusont (from the Thai Department of Communicable Disease Control) and colleagues now say that those who have acquired the infection by heterosexual intercourse are predominantly infected with the A serotype of HIV-1 (to the extent of 92 per cent), while those whose infection was acquired by intravenous injection were more likely (74 per cent) to be serotype B (The Lancet 345, 1078-1082;
1995). This finding supports the view that the rapid heterosexual spread of AIDS in Thailand is a function of a readily transmissible strain of HIV-1, presumably only recently introduced. That implies that the country's AIDS status is now in transition; serotype B will in due course be swamped by serotype A. And elsewhere? Should not sub-typing of HIV be routine?

The journal Applied Physics Letters is a very different kettle of fish, in reality the place in which to look for novel designs for semiconductor lasers, the devices that make our laser-printers work. But a group from Stanford University is worried by a more practical problem: how to know when the growing thickness of a layer of material being deposited on a silicon base has reached the thickness intended? J. Pei et al. have figured out that this can be done, in real time, simply by measuring the velocity of sound with vibration perpendicular to the growing silicon chip (Appl. Phys. Lett. 66, 2177-2179; 1995).

How can that be done? Simple. Support the silicon chip on two identical quartz rods pointed at the end, one of which is a source of ultrasound and the other is capable of receiving it. The transmitting rod is excited at $200 \mathrm{kHz}$ (quartz is piezoelectric) and generates an echo from the chip above, whose receipt can be recorded as a function of time. The difference between that time and that recorded by the second quartz rod gives the travel time along the surface of the chip. A simple experiment with a film being built up by sputtering shows that film thickness can be controlled to a small fraction of a micrometre.

Finally, in the New England Journal of Medicine is an encouraging account of a fetal brain tissue transplant into a patient suffering badly from Parkinson's disease (J. H. Kordower et al. 332, 1118-1124; 1995). The patient died of unrelated causes 18 months after transplantation, allowing those concerned to make a histochemical examination of his brain, and especially the substantia nigra, where dopamine appears to function. The conclusion is that the implanted neurons had survived "robustly". Although the patient had improved after the operation, he nevertheless required medication: he had not been cured but these are early days.

So there is a rag-bag of incidental information, but also a browser's charter. Nobody should complain that there are all those journals.

John Maddox 